\title{
La Responsabilidad Social Universitaria y su implementación: una revisión panorámica
}

\author{
University Social Responsibility and its implementation: a scoping review
}

\author{
Guadalupe del Carmen Olvera León $\bowtie$ \\ Universidad Autónoma de San Luis Potosí \\ San Luis Potosí, México
}

Omar Sánchez-Armáss Cappello

Universidad Autónoma de San Luis Potosí

San Luis Potosí, México

Oscar Alejandro Palacios Rodríguez

Universidad Autónoma de San Luis Potosí

oscar.palacios@uaslp.mx

San Luis Potosí, México

\section{Sayra Yesenia Medina Orta}

Universidad Autónoma de San Luis Potosí

San Luis Potosí, México

\section{Roxana Elizabeth Armendáriz Zarazua \\ Universidad Autónoma de San Luis Potosí \\ San Luis Potosí, México}

$\bowtie$ Autor por correspondencia

\begin{abstract}
Resumen:
El presente tuvo como objetivo explorar las investigaciones empíricas recientes sobre la Responsabilidad Social Universitaria (RSU). Se realizó una revisión de literatura basada en el diseño de scoping review de Arksey y O'Malley. Los resultados muestran que la mayoría de los estudios han sido publicados en Latinoamérica, en español y abordan temas principalmente relacionados al concepto, la enseñanza y los problemas de la RSU. La literatura muestra que el concepto de RSU ha incorporado diversas dimensiones que integran, particularmente, el cuidado del medio ambiente. Si bien la mayoría de los estudios se realizaron desde una aproximación cualitativa, las alternativas metodológicas utilizadas han sido diversas, de manera que no hay consenso sobre aquella que se defina como la más pertinente. La evaluación de la RSU al interior de las universidades, debe tener como base datos empíricos que permitan tomar decisiones respecto a sus funciones principales: la docencia, la investigación y la extensión; pues las problemáticas sociales actuales demandan soluciones que tengan como base los principios de la RSU en el trabajo conjunto de las universidades con la comunidad y otros sectores.
\end{abstract}

Palabras clave: Responsabilidad social; universidad; literatura; revisión; educación superior.

\begin{abstract}
:
The purpose of this study was to explore the recent empirical research on University Social Responsibility (USR). A literature review was performed following Arksey \& O'Malley's scoping review design. Results show that most studies have been published in Latin America, in Spanish and address the concepts, teaching and problems related with USR. The literature review shows the USR concept has incorporated a myriad of dimension, particularly integrating environmental care. Even though most studies were qualitative, diverse methodological approaches were employed, so that there is no consensus on what is defined as the most relevant. The USR evaluation within universities should be based on empirical data that allows for decision making in terms of teaching, research and outreach, considering that the current social issues demand solutions based on URS principles and a joint effort from universities, communities and other sector.
\end{abstract}

Keywords: Social responsibility; universities; literature; revision; higher education.

DOI:

https://doi.org/10.19136/etie.a4n8A.4756

Recibido: $\quad$ Aceptado: Publicado:

30/09/2021 15/12/2021 05/01/2022 


\section{La Responsabilidad Social Universitaria y su implementación: una revisión panorámica}

\section{| Introducción}

El concepto de Responsabilidad Social (RS) en las organizaciones, ha permitido gestionar los impactos provocados por éstas en el medio ambiente y la sociedad (Unión de Responsabilidad Social Universitaria Latinoaméricana [URSULA], 2019a). En el caso del ámbito universitario, se ha hablado del concepto de Responsabilidad Social Universitaria (RSU), cuyo origen se da en América Latina en la década de los años 2000. En este sentido, se ha considerado a la RSU como la evolución del concepto de RS; no obstante, la primera no se limita a la aplicación de la segunda en un ámbito universitario (Vallaeys, 2014).

La RSU propone que las universidades deben gestionar el impacto que tiene origen en la propia institución y su personal (Vallaeys \& Carrizo, 2006; Vallaeys et al., 2009; Vallaeys \& Álvarez, 2019). Igualmente, trata de promover la reflexión y el análisis sobre cómo la universidad responde a las necesidades económicas, políticas y sociales de la población; es decir, busca la conexión entre la universidad y su contexto próximo (Vallaeys et al., 2009). Las universidades que se preocupan por su entorno y/o contexto inmediato, pueden considerarse como socialmente responsables. Esto implica no solo crear conciencia de los actos y sus consecuencias directas, sino también de los impactos que tienen las acciones en el contexto social (Vallaeys, 2014).

Estas intenciones de gestión institucional se proponen a partir del conocido modelo URSULA, el cual abona a la consolidación del marco teórico latinoamericano de la RSU, por medio de diversas estrategias para la evaluación, el diseño, la implementación y la validación de prácticas en pro de la RSU. En este modelo de carácter heurístico, se pueden observar cuatro procesos de las Instituciones de Educación Superior (IES): (a) la formación, la cual parte de la metodología del aprendizaje basado en desafíos sociales; (b) la investigación, planteada desde la perspectiva de la investigación-acción realizada en y con la comunidad; (c) la extensión, que busca integrarse a la currícula y no como una práctica aislada y, finalmente; (d) la gestión, la cual considera que las políticas de la RSU se apliquen de manera transversal en la universidad (URSULA, 2019a).

A su vez, mediante estos procesos se proponen 12 metas de desempeño socialmente responsable que aportan hacia el logro de tres objetivos: (a) primero, la constitución de un marco temático explicativo de cómo transitar de la perspectiva proyectista y extensionista de las universidades hacia una perspectiva de transversalidad responsable; (b) segundo, la generación de pautas claras para la realización de autodiagnósticos institucionales sobre las percepciones de las y los universitarios sobre el ejercicio de la RSU y; (c) tercero, la posibilidad de concretar las prácticas socialmente responsables al interior de las cuatro funciones sustantivas de las IES (URSULA, 2019a).

De esta manera, el enfoque de la RSU ha sido aceptado año con año no solamente en universidades de América Latina, sino también de España (Vallaeys, 2014). En consecuencia, la RSU ha pasado a ser uno de los temas prioritarios en la agenda universitaria latinoamericana (Vallaeys, 2016).

Puesto que la construcción de nuevos conocimientos es uno de los objetivos de las universidades, y la investigación en RSU posibilita entender el impacto que generan 
los actos de la comunidad universitaria, la revisión de literatura se muestra como un medio pertinente para explorar lo que se ha establecido empíricamente en el campo de la RSU.

Así pues, se han realizado investigaciones de carácter bibliográfico y documental sobre el tema. Por una parte, URSULA (2019b) ha realizado el diagnóstico continental sobre el estado del arte de las IES adheridas a la unión. Por otra parte, estudios han analizado documentos nacionales e internacionales sobre la RSU (Casilla \& Camacho, 2012); además de las estrategias comunicativas (Cea Esteruelas, 2018) y educativas que implementan las propias universidades (Evia Alamilla et al., 2017; López Noriega et al., 2016). Asimismo, se han revisado metodologías en particular y su relación con la RSU (Martínez et al., 2019). No obstante, hay pocas revisiones que analizan los estudios empíricos en RSU (Duque \& Cervantes Cervantes, 2019; Olarte-Mejía \& Ríos-Osorio, 2015) y, particularmente, exploren los proceso que propone URSULA. De igual forma, las revisiones han dejado de lado la búsqueda en base de datos como Scielo y Redalyc, las cuales son referentes importantes para Latinoamérica; contexto donde surge el concepto de RSU.

Para comprender el alcance de la RSU, es relevante sistematizar los resultados y los hallazgos que han obtenido las investigaciones planteadas desde esta perspectiva. Por consiguiente, la presente revisión tiene como objetivo responder a la pregunta ¿Qué se ha investigado empíricamente sobre la RSU en la literatura actual?

Tabla 1

Palabras clave utilizadas en la búsqueda de literatura

\section{| Método}

Se llevó a cabo una revisión de literatura con base en el diseño de Scoping Review de Arksey \& O’Malley (2005), el cual posibilita realizar una exploración sobre el alcance de la literatura científica en un tema en particular; a través del análisis y resumen de literatura actual, se identifican áreas específicas para desarrollar investigaciones posteriores. Elaborar un Scoping Review sucede en cinco etapas: (a) identificación de una pregunta de investigación, (b) identificación de los estudios importantes, (c) selección de los estudios, (d) extracción de los datos y (e) recopilación, síntesis y comunicación de los resultados. De igual forma, se consultó la lista de verificación Preferred Reporting Items for Systematic reviews and Meta-Analyses extension for Scoping Reviews (PRISMA$\mathrm{ScR}$ ) para el desarrollo del presente documento (Tricco et al., 2018).

Para la búsqueda de literatura se consultaron los índices y las bases de datos de Scielo, Redalyc, PubMed, EBSCO y Scopus. Las palabras clave fueron adecuadas al estilo del buscador de cada uno de los sitios consultados; además de utilizar los términos booleanos y las opciones avanzadas para mejorar la búsqueda.

El acceso y la descarga de los trabajos fue en su totalidad a través de acceso libre. En la Tabla 1 se presentan las palabras clave que permitieron obtener un mayor éxito en los sitios revisados.

\begin{tabular}{cccc}
\hline Índice/Base de datos & Palabras clave utilizadas & Combinación utilizada & Resultados \\
\hline PubMed & $\begin{array}{c}\text { university social responsibility/ } \\
\text { social responsibility }\end{array}$ & $\begin{array}{c}\text { (university social responsibili- } \\
\text { ty[Title/Abstract]) NOT (social } \\
\text { responsibility[Title/Abstract]) }\end{array}$ \\
\hline EBSCO & $\begin{array}{c}\text { university social responsibility/ } \\
\text { social responsibility }\end{array}$ & $\begin{array}{l}\text { AB university social responsibi- } \\
\text { lity NOT AB social responsibility }\end{array}$ \\
\hline
\end{tabular}




\begin{tabular}{|c|c|c|c|}
\hline Índice/Base de datos & Palabras clave utilizadas & Combinación utilizada & Resultados \\
\hline Scopus & university/social/responsability & $\begin{array}{l}\text { ( TITLE-ABS-KEY ( university } \\
\text { AND social AND responsibility ) } \\
\text { AND NOT TITLE-ABS-KEY ( so- } \\
\text { cial AND responsibility ) ) }\end{array}$ & 0 \\
\hline Scielo & $\begin{array}{l}\text { responsabilidad social universi- } \\
\text { taria }\end{array}$ & $\begin{array}{l}\text { responsabilidad social universi- } \\
\text { taria }\end{array}$ & 97 \\
\hline Redalyc & $\begin{array}{l}\text { responsabilidad social universi- } \\
\text { taria }\end{array}$ & $\begin{array}{l}\text { responsabilidad social universi- } \\
\text { taria }\end{array}$ & 438 \\
\hline
\end{tabular}

Nota: Elaboración propia.

Cómo criterios de selección se estableció la revisión de artículos empíricos cuantitativos, cualitativos y mixtos; asimismo, se revisaron trabajos publicados en inglés, español y portugués.

Los artículos fueron elegidos y descartados de acuerdo con que el título, el resumen y el texto completo cumplieran los criterios mencionados anteriormente. Se tuvo acceso a un total de 131 artículos, de los cuales se recuperaron todos en texto completo. De estos, 55 no fueron considerados por el título y 12 más fueron excluidos por resumen, ya que obedecían a trabajos teóricos y revisiones. Al ser leídos los trabajos, se descartaron 13 más, lo que dejó un total de 51 artículos para ser sistematizados y analizados (Figura 1).

Figura 1

Diagrama sobre la selección de la literatura

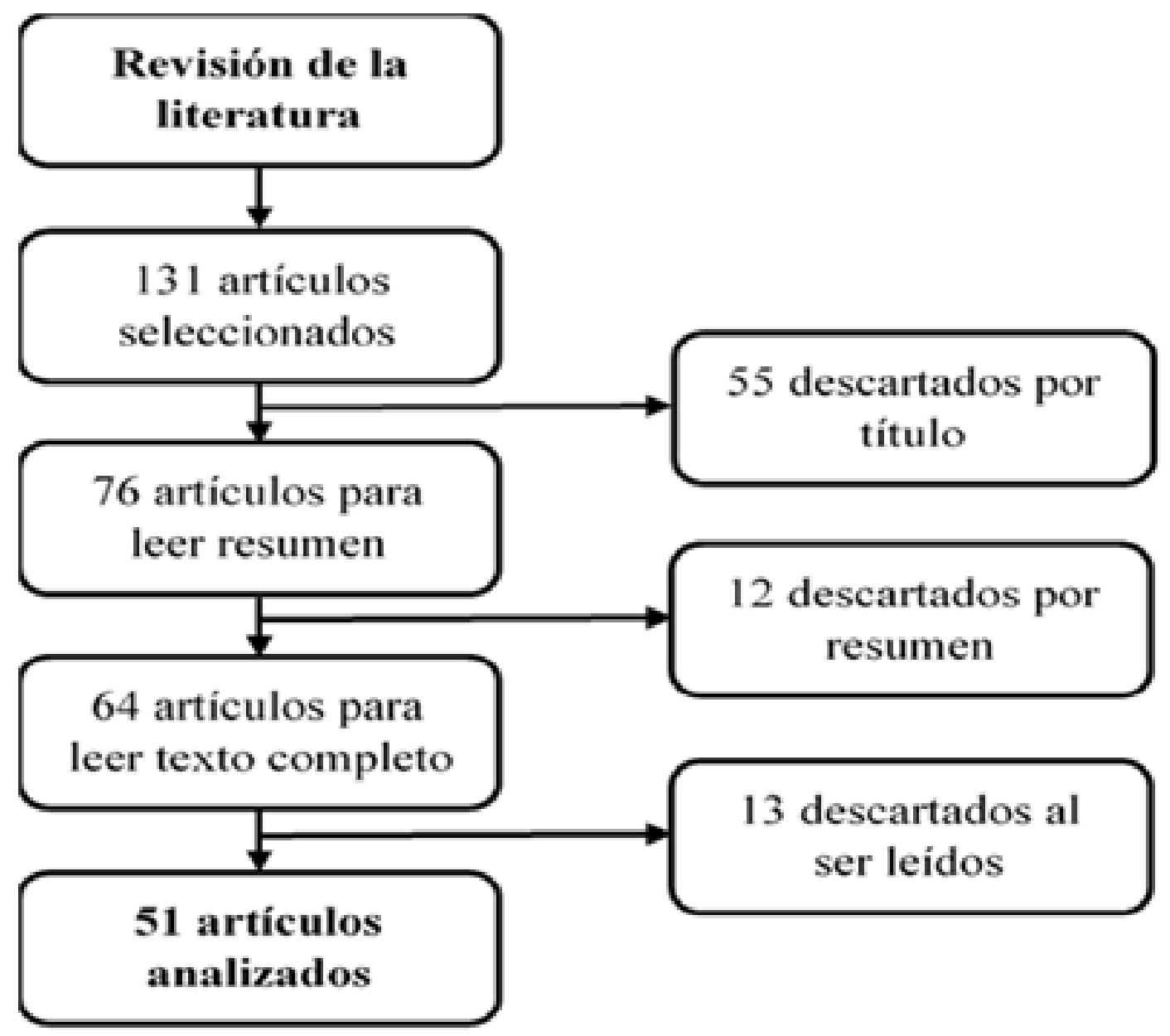

Nota: Se muestra el proceso para la inclusión y la eliminación de literatura para su posterior lectura y análisis. 
Los trabajos elegidos y leídos en texto completo se sistematizaron en una tabla matriz que incluía los siguientes datos: autores, año, enfoque, diseño, lugar, muestra, instrumentos o técnicas, variables o dimensiones, análisis y resultados principales. Por último, los resultados de cada trabajo fueron analizados y agrupados en áreas temáticas (Arksey \& O’Malley, 2005; Popay et al., 1995).

Tabla 2

Características generales de los estudios

\section{| Resultados}

Del total de 51 artículos leídos, sistematizados y analizados, se encontró que la mayoría de ellos fueron escritos en español y en Colombia. El enfoque metodológico más reportado fue el cualitativo, a la vez que el diseño más utilizado fue el descriptivo. Gran parte de los estudios realizados consideraron la participación de toda la comunidad universitaria. En cuanto al año, los artículos más antiguos fueron del 2008; mientras que los más actuales fueron del 2020 (Tabla 2).

\begin{tabular}{|c|c|c|c|}
\hline Característica & Número & Característica & Número \\
\hline $\begin{array}{l}\text { Idioma } \\
\text { Español } \\
\text { Inglés }\end{array}$ & $\begin{array}{c}49 \\
2\end{array}$ & $\begin{array}{c}\text { Enfoque } \\
\text { Cualitativo } \\
\text { Cuantitativo } \\
\text { Mixto }\end{array}$ & $\begin{array}{c}24 \\
22 \\
5\end{array}$ \\
\hline $\begin{array}{c}\text { País } \\
\text { Colombia } \\
\text { Venezuela } \\
\text { Chile } \\
\text { México } \\
\text { España } \\
\text { Ecuador } \\
\text { Perú } \\
\text { Cuba } \\
\text { España, Chile, Colombia y Perú } \\
\text { Paraguay } \\
\text { Puerto Rico } \\
\text { Uruguay }\end{array}$ & $\begin{array}{c}10 \\
9 \\
8 \\
8 \\
7 \\
2 \\
2 \\
1 \\
1 \\
1 \\
1 \\
1\end{array}$ & $\begin{array}{c}\text { Diseño } \\
\text { Descriptivo } \\
\text { Estudio de caso } \\
\text { Exploratorio } \\
\text { Correlacional } \\
\text { Hermenéutico } \\
\text { Teoría fundamentada } \\
\text { Aprendizaje servicio } \\
\text { Explicativo } \\
\text { Etnografía } \\
\text { Investigación acción }\end{array}$ & $\begin{array}{l}22 \\
11 \\
7 \\
3 \\
2 \\
2 \\
1 \\
1 \\
1 \\
1\end{array}$ \\
\hline $\begin{array}{c}\text { Participantes } \\
\text { Comunidad universitaria } \\
\text { Estudiantes } \\
\text { Stakeholders } \\
\text { Directivos } \\
\text { Personas beneficiarias } \\
\text { Docentes } \\
\text { Personal universitario } \\
\text { Estudiantes y docentes } \\
\text { Estudiantes y personas egresadas } \\
\text { Personas coordinadoras y beneficia- } \\
\text { rias de programas } \\
\text { Estudiantes y personas beneficiarias }\end{array}$ & $\begin{array}{c}21 \\
10 \\
7 \\
3 \\
2 \\
2 \\
2 \\
1 \\
1 \\
1\end{array}$ & $\begin{array}{l}\text { Año } \\
2015 \\
2019 \\
2018 \\
2016 \\
2017 \\
2014 \\
2012 \\
2020 \\
2011 \\
2008 \\
2013 \\
2010 \\
2009\end{array}$ & $\begin{array}{l}8 \\
7 \\
6 \\
6 \\
4 \\
5 \\
5 \\
3 \\
2 \\
2 \\
1 \\
1 \\
1\end{array}$ \\
\hline
\end{tabular}

Nota: Elaboración propia. 
Los temas reportados con mayor frecuencia por los estudios son presentados y narrados en tres áreas temáticas: concepto de la RSU, enseñanza de la RSU y problemas de la RSU. A su vez, éstas se dividen en otras subáreas (Tabla 3).

Tabla 3

Áreas temáticas reportadas con mayor frecuencia en los estudios seleccionados

Áreas Concepto de RSU

Estudio
Enseñanza de la RSU

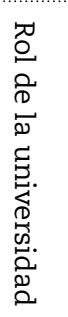

Problemas de la RSU

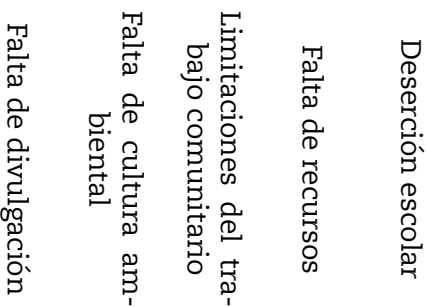

Ahumada et al. (2018)

Aldeanueva y Jiménez (2013)

Alonzo - Godoy (2016)

Alvarado et al. (2017)

Arango et al. (2014)

Aristimuño y Rodríguez

(2014)

Ayala et al. (2018)

Céspedes (2019)

Chirinos y Pérez (2016)

Corretgé y Miret (2018)

Díaz y Facal (2011)

Gaete (2008)

Gaete (2010)

Gaete (2011)

Gaete (2014)

Gaete (2015) planes

Gaete (2015) significados

Gaete (2015) stakeholders

Gaete (2015) voluntariado

Gaete (2016)

Giménez et al. (2016)

Gómez et al. (2018)

Huerta y Gaete (2017)

Ibarra et al. (2020)

Lescher et al. (2015)

López - Noriega (2016)

Lúquez et al. (2012)

Madorrán - García (2012)

Malluk y D’Luyz (2018)

Martí et al. (2014)

Martínez et al. (2008)

Mendoza et al. (2019)

Moreno y Aguirre (2018)

Nuñez (2015)

Nuñez et al. (2020)

Pepe et al. (2009) 


Pepe et al. (2009)
Peralta (2012)
Pumacayo (2020)
Ramos (2012)
Rangel et al. (2012)
Raza y Loachamín (2015)
Rojas et al. (2020)
Tobón et al. (2019)

Nota: Elaboración propia.

\section{Concepto de RSU}

La definición de la RSU en los estudios se muestra relacionada principalmente con aspectos éticos y cívicos, así como a los valores y los principios de la universidad (Gaete Quezada, 2014, 2015a). Asimismo, se encuentra asociada al quehacer universitario en aspectos como la docencia, la investigación, la extensión y la gestión, las cuales afirman deben desarrollarse a través de un ejercicio transparente (Gaete Quezada, 2015a).

Por su parte, tanto los puestos directivos como las partes interesadas, definen el comportamiento socialmente responsable a partir de su impacto, las respuestas y los esfuerzos del mismo quehacer universitario (Gaete Quezada, 2015a, 2015c).

Algunos estudios proponen dimensiones que conforman el concepto de RSU, dentro de estos se encuentran el cuidado del medio ambiente (Ahumada-Tello et al., 2018; Aldeanueva Fernández \& Jiménez Quintero, 2014), la preocupación social y económica (Aldeanueva Fernández \& Jiménez Quintero, 2014; Arango Tobón et al., 2014), la generación y aplicación del conocimiento
(Alvarado Lagunas et al., 2017; Céspedes Aguirre, 2019), la autotrascendencia (Martí Noguera et al., 2014), la cohesión social, el respeto a la diversidad (Gaete Quezada, 2010), la rendición de cuentas (Gaete Quezada, 2015a), el campus responsable y la formación profesional ciudadana (Ahumada-Tello et al., 2018).

Dentro de la definición y las dimensiones de la RSU, sobresale el cuidado del medio ambiente. De esta forma, algunas instituciones universitarias reportan haber implementado estrategias para el reciclaje con el propósito de ser menos perjudiciales y respetar el medio ambiente (Aldeanueva Fernández \& Jiménez Quintero, 2014; Méndoza Avilés et al., 2019; Raza Calderón \& Loachamín Paucar, 2015; Velasco Ramírez et al., 2017).

Dichas estrategias son percibidas por la comunidad universitaria como perfectamente viables y han mostrado una alta participación por parte de la misma comunidad (Díaz Molina \& Facal Santiago, 2011; Pepe et al., 2009; Raza Calderón \& Loachamín Paucar, 2015; Uribe Macías et al., 2016). Así pues, se propone que estas estrategias no solo deban ser realizadas por ciertas carreras afines, sino por toda la comunidad perteneciente a la universidad (Rangel 
Toloza et al., 2012).

El cuidado del medio ambiente como dimensión de la RSU se percibe con mayor frecuencia en las universidades, por lo que la misma es contemplada dentro de sus valores y sus principios (Gaete Quezada, 2010; Uribe Macías et al., 2016). Esto ha llevado a que algunas universidades hayan organizado comités para el cuidado del medio ambiente, los cuales se caracterizan por la presencia tanto de agentes internos como externos (Gaete Quezada, 2014).

\section{Enseñanza de la RSU}

Según los artículos revisados, la RSU es presentada como una parte importante dentro de la enseñanza en la universidad, por lo que la misma es considerada como un componente relevante para los valores institucionales y los procesos de aprendizaje (Alvarado Lagunas et al., 2017; Ayala Palacios et al., 2018; Gaete Quezada, 2015; Giménez et al., 2016; Lúquez de Camacho et al., 2012; Peralta, 2012; Rangel Toloza et al., 2012).

De esta manera, se considera que la RSU tiene una gran trascendencia para los principios universitarios (Alvarado Lagunas et al., 2017; Gaete Quezada, 2008), la enseñanza académica (Alvarado Lagunas et al., 2017; Ayala Palacios et al., 2018; Díaz Molina \& Facal Santiago, 2011), el plan de estudios (Vásquez Ibáñez, 2019), el ejercicio profesional (Ayala Palacios et al., 2018; Rangel Toloza et al., 2012), la participación social (Alonzo-Godoy et al., 2016; Gaete Quezada, 2008), el impacto ambiental y el desarrollo sustentable (Ayala Palacios et al., 2018; Gaete Quezada, 2008).

La integración de la RSU ha permitido que los procesos de enseñanza en algunas universidades sean más cercanos a la comunidad (Gaete Quezada. 2008, 2015a; Moreno Moreno \& Aguirre Gonález, 2018; Rangel Toloza et al., 2012). En este sentido, se ha reportado que universidades han ido incorporando los principios de la RSU a sus planes y sus estrategias de enseñanza (Gaete Quezada, 2015d; Huerta-Riveros \& Gaete-Feres, 2017; Madorrán-García, 2012; Raza Calderón \& Loachamín Paucar, 2015).

Los estudios refieren a la universidad como un espacio que ha alcanzado una relevancia social importante, lo que ha posibilitado crear ámbitos externos de gestión para el quehacer universitario, así como estrategias de servicios en la comunidad (Gaete Quezada, 2015c; Malluk Marenco \& D’Luyz Sánchez, 2017; Ramos Parra, 2012).

La universidad se presenta como un espacio de aprendizaje para nuevos conocimientos y un lugar sustentable, donde se procura el respeto a la comunidad y se mantiene cercanía a las necesidades de esta última (Alvarado Lagunas et al., 2017; Gaete Quezada, 2008, 2010). $\mathrm{Al}$ ser un espacio para el aprendizaje, se considera como una instancia para la formación de estudiantes con un sentido de compromiso y contribución al desarrollo de la sociedad (Gaete Quezada, 2008).

A su vez, se menciona que la universidad debe promover la inclusión y dejar de lado la exclusión, fomentar la investigación y la cultura, además de promover la transparencia (Gaete Quezada, 2008, 2015c). Por consiguiente, algunas universidades han optado por la contratación de proveedores locales, puesto que ha sido relacionado a una de las tareas de la RSU (Gaete Quezada, 2014).

La divulgación y la promoción del quehacer universitario es vista como una estrategia básica para la vinculación con distintos sectores públicos y privados (Martínez de Carrasquero et al., 2008). En consecuencia, algunas universidades han optado por difundir mediante sus páginas web las memorias de eventos académicos; asimismo, se han utilizado espacios como los programas de radio en los cuales se trata de dar voz a grupos 
desfavorecidos o en situación vulnerable (Gaete Quezada, 2014).

La noción de universidad pública se relaciona a un espacio para el acceso a la información y el conocimiento por parte de toda la sociedad; además de una instancia para la generación de investigaciones recientes que busquen el beneficio regional. De esta manera, las estrategias de extensión son percibidas como cuestiones fundamentales para un quehacer universitario cercano a las necesidades del entorno que rodea a la misma universidad (Gaete Quezada, 2015c).

Se ha encontrado que el rol de lasy los docentes es una parte importante para la RSU. Dicho rol se ha visto enriquecido por el compromiso y la motivación, lo cual conlleva al fortalecimiento de las competencias estudiantiles (Gaete Quezada, 2014). Asimismo, la participación, el liderazgo, la investigación y la autogestión han sido mencionadas como parte importante del rol y la función docente (Pepe et al., 2009). En consecuencia, docentes que realizan estrategias de reforzamiento positivo en la enseñanza, suelen convertirse en figuras de refuerzo para las y los estudiantes (Peralta, 2012).

Se ha reportado en algunas universidades que las y los docentes que muestran un alto grado de pertenencia a la universidad, tienen un fuerte compromiso con las dimensiones de la RSU, lo que hace que contextualicen y transmitan de mejor manera el conocimiento (Vera Guadrón et al., 2012). De esta forma, las y los docentes que refieren un fuerte sentimiento de relación con la universidad, suelen mostrar un mejor desempeño (Rangel Toloza et al., 2012).

Por esta razón, se ha buscado que el personal docente sea promotor de actividades para el servicio y la responsabilidad social (Peralta, 2012); pues los puestos directivos se encuentran conscientes de la relevancia que tienen las y los docentes en la formación de personas profesionales comprometidas con la sociedad, la naturaleza y la democracia (Raza Calderón \& Loachamín Paucar, 2015).

Aunado al rol de la universidad, se encuentra la evaluación de ésta. Distintas partes que conforman la comunidad han evaluado a las universidades, principalmente de forma negativa, por lo que se refieren varios aspectos a mejorar.

Por una parte, las y los docentes consideran necesario, dentro de la universidad, mejorar los procesos para la integración de actoras y actores sociales de la comunidad (Aristimuño \& Monroy, 2014). Por otra parte, la RSU en la universidad ha sido calificada por las y los estudiantes como aceptable; no obstante, consideran que la misma no genera espacios e instancias que busquen la solidaridad entre estudiantes (Díaz Molina \& Facal Santiago, 2011; López Noriega et al., 2016).

A su vez, ha habido personas que consideran que las universidades no se vinculan con la sociedad, no divulgan el conocimiento y por ende no se dan a conocer (Céspedes Aguirre, 2019). En consecuencia, se percibe que la universidad parece haberse centrado solo en los procesos y las preocupaciones internas, lo que ha provocado que estudiantes perciban a la misma con tensión y crítica (Gaete Quezada, 2011, 2015b).

Parte importante de quienes se enfocan en el quehacer universitario y evalúan el mismo, son las personas interesadas o stakeholders. Por personas interesadas se ha incluido a quienes emplean, estudiantes, sindicatos, organizaciones estudiantiles, gobiernos locales y regionales, sociedades civiles, docentes, aspirantes, personas estudiantes extranjeras, egresadas y donantes (Céspedes Aguirre, 2019; Gaete Quezada, 2011). 
Los estudios han explorado sobre la inclusión de las partes interesadas de la RSU en la universidad, además de la manera en cómo se reflejan éstas en sus misiones y valores (Gaete Quezada, 2010). Por lo tanto, se ha visto que las universidades tratan de mantenerse en cercanía con las y los stakeholders a través de medios como el internet (Gaete Quezada, 2011).

\section{Problemas de la RSU}

Ahora bien, en las universidades también se ha presenciado la carencia del concepto de RSU en sus valores y sus principios (Gaete Quezada, 2014, 2016; Ibarra Uribe et al., 2020; Vásquez Ibáñez, 2019); de modo que los cursos relacionados a la RSU se muestran ausentes en los planes de estudio (Céspedes Aguirre, 2019; Vásquez Ibáñez, 2019). Esto lleva a una formación deficiente en materia de RSU en sus estudiantes, lo cual es referido por las y los mismos (Peralta 2012; Pumacayo Palomino et al., 2019).

Igualmente, se ha encontrado que en las instituciones universitarias hace falta la presencia de centros o departamentos encargados de la gestión y del servicio de la RSU (Aldeanueva Fernández \& Jiménez Quintero, 2014; Gaete Quezada, 2014; Ramos Parra, 2012).

La falta de RSU se ve reflejada en la escasez de planes de estudio que aborden el tema y la ausencia de centros encargados de su gestión, lo que lleva a tener un conocimiento de la comunidad lejano a la realidad $\mathrm{y}$, en consecuencia, acciones como la participación, la investigación y la vinculación con la misma comunidad son limitadas (Céspedes Aguirre, 2019; Gaete Quezada, 2008; Raza Calderón \& Loachamín Paucar, 2015; Rojas Valdés et al., 2020).

La ausencia de acciones para la divulgación del trabajo realizado en las universidades es algo que frecuentemente es encontrado en los estudios. Si bien hay eventos de difusión donde se exponen los resultados de las investigaciones, no hay una participación de los grupos de interés. A pesar de que es común el trabajo de campo, no hay una rendición de cuentas a las personas beneficiarias; pues los resultados se muestran en eventos dirigidos primordialmente al público académico (Céspedes Aguirre, 2019).

Aunque las universidades cuentan con sitios web y redes sociales donde se trata de divulgar el quehacer universitario, se ha considerado que hace falta un mayor acercamiento a la población en general (Gaete Quezada, 2011).

Se observó que las universidades también carecen de estrategias, principios y gestiones que busquen una cultura ambiental; o en su caso, cuando se afirma tenerlas, no se perciben acciones concretas que promuevan las mismas o que tengan un gran impacto (Alonzo-Godoy et al., 2016; Céspedes Aguirre, 2019; Gómez et al., 2018; Méndoza Avilés et al., 2019; Velasco Ramírez et al., 2017).

El desconocimiento de estas estrategias es común en la comunidad universitaria. En algunos casos, las acciones están en dependencia del alumnado, limitándose al aspecto del reciclaje, por lo que la participación institucional es pobre y limita la adquisición de hábitos ecológicos (Alonzo-Godoy et al., 2016; Díaz Molina \& Facal Santiago, 2011; Pumacayo Palomino et al., 2019; Uribe Macías et al., 2016).

Estudios reportaron limitaciones por parte de las universidades para ofrecer servicios y realizar trabajo en campo y comunidades. Algunas limitaciones mencionadas fueron la resistencia al cambio por parte de docentes y la falta de recursos en las instituciones. Respecto a esto último, se menciona que los recursos son financiados mayoritariamente por la universidad, en 
una parte por las y los mismos estudiantes y una escasa aportación es de otras instituciones (Rangel Toloza et al., 2012).

La dificultad del trabajo en la comunidad se ha visto influenciada a su vez por la poca gestión y la falta de coordinación con líderes y profesionales pertenecientes a la misma. Esto ha contribuido a que el trabajo se desarrolle de manera desarticulada y con falta de consenso. Aunado a esto, cabe considerar la poca preparación, la desmotivación y el escaso apoyo de instituciones (Rojas Valdés et al., 2020).

Dada la poca presencia de la universidad en la comunidad, las visitas de equipos de trabajo universitarios son percibidas como extrañas y con desconfianza, lo que en ocasiones ha llevado a rechazar el trabajo ofrecido por parte de la misma (Rojas Valdés et al., 2020).

Asimismo, la existencia de estructuras en gran parte centralizadas, ha dificultado la participación y la comunicación con la comunidad (Lescher et al., 2015). En otros casos, el trabajo se ve limitado por el propio desinterés estudiantil en la comunidad, dado que la mayoría suele interesarse por realizar sus prácticas profesionales en otros campos o contextos (Tobón Marulanda et al., 2019).

Se considera que la falta de recursos es una limitante importante para la implementación de estrategias en RSU (Nuñez Flores et al., 2020). Esto ha sido particularmente un obstáculo en las y los estudiantes que tienen la intención de emprender iniciativas o negocios (Chirinos Araque \& Pérez Peralta, 2017; Vega et al., 2018).

Este problema también se encuentra sumamente relacionado a la deserción escolar en estudiantes (Zárate Rueda \& Mantilla Pinilla, 2014). Dada las limitaciones económicas, una gran parte de estudiantes tenía que trabajar, lo que limitaba los tiempos para dedicar a las actividades de la escuela (Zárate-Rueda \& HernándezSuárez, 2016).

Una de las problemáticas que fueron analizadas desde la RSU, fue el abandono escolar. Razones que motivaron el abandono por parte de estudiantes fueron los paros y los cambios de fecha en ciclo escolar, la falta de comunicación con profesores y la ausencia de metodologías para las diversas modalidades de enseñanza. Otra más fue los problemas económicos, lo que obligaba a estudiantes a trabajar y a su vez influía en un bajo rendimiento académico, dado el poco tiempo que se contaba para cumplir con las obligaciones. Asimismo, otras causas identificadas fueron los embarazos no planeados y el padecimiento de enfermedades que impedían continuar con los estudios (Zárate-Rueda \& Hernández-Suárez, 2016; Zárate Rueda \& Mantilla Pinilla, 2014).

\section{| Discusión}

La revisión de la literatura permitió definir que los estudios realizados desde la RSU se han publicado principalmente en español y en Latinoamérica, dado que el concepto tuvo sus orígenes en esta región (Vallaeys, 2014). Si bien la mayoría de estudios fueron desde una aproximación cualitativa, las alternativas metodológicas utilizadas han sido diversas, de manera que no hay consenso sobre aquella que se defina como la más pertinente.

En cuanto al periodo temporal, aunque el concepto de RSU surge en el año 2000 (Vallaeys, 2014), fue hasta el año 2008 que comienzan a reportarse los primeros estudios que presentaban resultados propios de la investigación. Los estudios en su mayoría fueron realizados contemplando como muestra la comunidad universitaria en su totalidad.

El análisis de la literatura mostró una diversidad de temas, aunque se pudo encontrar que los más recurrentes obedecen al concepto, la enseñanza y los problemas 
de la RSU. El concepto de RSU se encontró relacionado a la ética y la cívica, particularmente en lo relacionado con los principios y el quehacer de la universidad. Esto puede observarse en algunas dimensiones que forman el concepto de RSU y pueden enmarcarse en los principales ámbitos de acción de la RSU (Vallaeys et al., 2009).

Respecto a lo encontrado en la literatura, el cuidado del medio ambiente, la autotrascendencia, la cohesión social y el respeto por la diversidad son aspectos que pueden integrarse a los vínculos sociales que las IES tejen con diversos actores de su entorno. La preocupación social y económica, la rendición de cuentas y el campus responsable pueden formar parte de la gestión organizacional de las IES. La generación y aplicación del conocimiento y la formación profesional ciudadana tienen que ver con los conocimientos que producen y la formación que brindan las IES (Vallaeys et al., 2009).

Una de las dimensiones más referida en las investigaciones fue lo relacionado al cuidado y mantenimiento del medio ambiente. Si bien esto forma parte importante de la RS de cualquier institución pública o privada, la gran mayoría de los estudios desde la RSU han dirigido los esfuerzos particularmente a esta dimensión, por lo que solo se atienden a un aspecto parcial de lo que realmente representa la RSU con base en sus ámbitos y metas (URSULA, 2019a). En este sentido, cabe considerar que el cuidado del medio ambiente no solo se remite al entorno físico y al acceso de recursos naturales, como fue referido en varios estudios revisados, sino que involucra también el cuidado del ambiente social y cultural (Corral Verdugo, 2010).

Así pues, para hablar de una universidad sustentable y preocupada por el medio, debe considerarse un cambio en los modelos y la participación de toda la comunidad universitaria; puesto que se ha encontrado que este tipo de iniciativas son difíciles de mantener a lo largo del tiempo, de manera que puedan obtenerse cambios perceptibles. Es por esto que, las iniciativas en materia del cuidado del ambiente físico y social, requieren de la conformación de comunidades universitarias sensibles a las necesidades ambientales actuales y se comprometan al manejo de las consecuencias de las mismas (Gaudiano et al., 2015). De esta manera, cabría indagar sobre hasta qué punto es incluido en los principios y los valores universitarios la preocupación por el cuidado del medio ambiente.

Debe considerarse que las universidades también generan y reproducen impactos negativos en las sociedades y sus contexto inmediatos; por consiguiente, es importante la evaluación constante de las universidades (Vallaeys, 2014). Sin embargo, la literatura se ha limitado a evaluar solo la calidad de la enseñanza y la academia (Gálvez \& Gutiérrez, 2018), lo que evidencia la falta de investigación sobre cómo es que la sociedad percibe a las universidades en materia de sus impactos, cómo es que las evalúa y como es que, inclusive, se ha llegado a percibir con cierta desconfianza a la misma (Flores-Garrido \& Barreto-Ávila, 2018).

La generación de estrategias para la evaluación y el seguimiento durante la trayectoria académica de estudiantes, es relevante para que se garantice una formación y un rol más comprometido con la sociedad y sus problemáticas actuales (Evia Alamilla et al., 2017). Es importante que se cuestionen los marcos que subyacen al quehacer universitario y se integren dentro de los planes de estudios asignaturas que aborden la RSU. No obstante, se debe reconocer que el transmitir solo información no basta para que las universidades se muestren fuertemente sustentadas en la realidad social inmediata.

De igual forma, el rol de las y los profesores universitarios es un elemento importante para la formación de estudiantes y su trascendencia dentro de la sociedad, dado que los primeros se muestran como modelo profesional 
y responsable de quien enseña y forma a las futuras personas profesionales (Garbizo Flores et al., 2020a).

Pensar en la RSU, implica ser conscientes de la manera en cómo las universidades se ven comprometidas con el trabajo comunitario. Las acciones universitarias deben de buscar acciones de cooperación conjunta con las personas beneficiarias, de modo que se refiera una participación genuina (Fernández Díaz, 2019). Esto permitirá no solo la transformación social de la comunidad, sino también la transformación al interior de la misma universidad (Garbizo Flores et al., 2020b). En este sentido, el trabajo de las universidades con otras instancias y sectores es de suma relevancia. Las acciones en conjunto con las autoridades, el Estado y el sector privado puede aportar soluciones colaborativas para la transformación de la sociedad y el desarrollo humano (López-Noriega et al., 2015).

La trascendencia sobre el trabajo de la universidad y su impacto en la solución de problemáticas actuales en la sociedad, puede verse en cambios estructurales que posibiliten el cumplimiento de la agenda del 2030 con relación a los Objetivos de Desarrollo Sostenible (ODS). La participación de la universidad es relevante desde su función de formar profesionales. Sin embargo, su papel no se limita a esto; pues cuenta con un rol primordial en la investigación y la generación de nuevos conocimientos, así como la creación de espacios de dialogo y debate a través de los cuales se posibilite promover valores y principios que busquen la transformación del mundo y lo hagan un lugar mejor (Cano Ginés, 2019).

La universidad con respecto a la generación de investigación tiene un rol importante en la divulgación del conocimiento; de manera que debe buscar los medios adecuados para que los resultados o los hallazgos de los estudios científicos lleguen a la sociedad en general. Cabe discutir sobre las limitaciones en las universidades que han fomentado poco el intercambio académico y la participación de sus estudiantes en eventos científicos, lo que representa una barrera importante para la divulgación de la ciencia (Carranza Esteban \& Turpo Chaparro, 2019).

A pesar de que la RSU se ha abierto paso en recientes años, aún falta que se integre un mayor número de universidades a esta perspectiva, de modo que se vea reflejada en términos de políticas institucionales. De forma similar, se ha encontrado en la actualidad que ninguna universidad cumple con los requisitos para ser considerara como una IES de excelencia en materia de RSU (URSULA, 2019b). Se observa un compromiso y avances en cuanto a la integración de la RSU en las universidades, aunque a la vez se necesita un mayor compromiso para que sea incorporada y forme parte importante de las políticas y las decisiones de las universidades.

$\mathrm{Al}$ respecto, se ha encontrado que de los aspectos que han sido mejor implementados en las universidades es la mejora del ambiente de trabajo; mientras que la docencia e investigación han sido considerados como aspectos urgentes en los cuales trabajar (URSULA, 2019b). Asimismo, resultados muestran que las universidades integran la RSU desde distintas perspectivas; pues se ha encontrado que las IES públicas buscan la mejora funcional y la relación al interior de sus grupos; mientras que las IES privadas se enfocan hacia el exterior en miras de mejorar su competitividad en el mercado (Olarte-Mejía \& Ríos-Osorio, 2015).

Así pues, las funciones de docencia, investigación y extensión dentro de las universidades deben de caracterizarse por la formación profesional socialmente responsable (Casilla \& Camacho, 2012). La evaluación de la RSU al interior de las universidades debe de verse reflejada en datos empíricos que permitan tomar decisiones al respecto, de modo que se le de atención a las problemáticas propias de la comunidad donde se 
encuentran insertas.

Responsabilidad Social Universitaria: Medición del impacto de una asignatura en estudiantes de En conclusión, la literatura muestra que el concepto de Ingeniería. Ingeniería. Revista Académica, 20(2), 76-84. RSU ha incorporado diversas dimensiones, donde se ha abordado particularmente el cuidado del medio ambiente. Las problemáticas actuales demandan soluciones y acciones desde distintas áreas del conocimiento, donde las universidades, mediante la integración de los principios de la RSU, garanticen la formación de personas profesionales más sensibles y cercanas a las necesidades propias del contexto inmediato. Para esto, es necesaria la participación conjunta con distintos sectores que faciliten dar respuestas a dichas necesidades, además de generar los espacios para el desarrollo de investigación y su potencial divulgación en miras del beneficio de la misma comunidad.

La presente revisión permitió explorar qué hallazgos empíricos se han encontrado en investigaciones planteadas desde la RSU; en consecuencia, solo fueron analizados artículos empíricos y no se incluyeron otros recursos como informes, libros, tesis y revisiones teóricas. Se recomienda darle continuidad a los resultados propios que se generan en investigación en materia de RSU, especialmente si se busca definir las aproximaciones metodológicas más adecuadas para su estudio.

\section{| Referencias}

Ahumada-Tello, E., Ravina-Ripoll, R. \& López-Regalado, M. E. (2018). Responsabilidad Social Universitaria. Desarrollo de competitividad organizacional desde el proceso educativo. Actualidades Investigativas En Educación, 18(3), 1-30. https://doi.org/h9rj

Aldeanueva Fernández, I. \& Jiménez Quintero, J. A. (2014). Responsabilidad social universitaria en España: un estudio de casos. Revista Venezolana de Gerencia, 18(64), 649-662. https://doi.org/jbgw

Alonzo-Godoy,M.,Ayora Herrera,E. \& Bote Caamal, R.(2016).

AlvaradoLagunas,E.,Morales Ramírez,D.\& Ortiz Rodríguez,

J. (2017). Un análisis sobre la percepción que los directivos y docentes tienen de la responsabilidad social universitaria en las facultades de contaduría y administración en México. Universidad \& Empresa, 19(32), 37-59. https://doi.org/g9fs

Arango Tobón, O., Clavijo Zapata, S., Puerta Lopera, I. \& Sánchez Duque, J. (2014). Formación académica, valores, empatía y comportamientos socialmente responsables en estudiantes universitarios. Revista de La Educacion Superior, 43(169), 89-105.

Aristimuño, M. \& Monroy R, C. (2014). Responsabilidad social universitaria. Su gestión desde la perspectiva de directivos y docentes. Estudio de Caso: Una pequeña universidad latinoamericana. Asociación Interciencia, 39(6), 375-383.

Arksey, H. \& O'Malley, L. (2005). Scoping studies: Towards a methodological framework. International Journal of Social Research Methodology, 8(1), 19-32. https://doi. org/bquqne

Ayala Palacios, R., Leyva C, O., Tamez G, G. \& Hernández P, A. (2018). Gestión universitaria y gestión de la investigación en la universidad: aproximaciones conceptuales. Revista Venezolana de Gerencia, 23(1), 131-145. https://doi.org/10.31876/revista.v23i1.24460

Cano Ginés, A. (2019). El papel de la Universidad en la Cooperación Internacional al Desarrollo. Los ODS y el horizonte 2030 [Ponencia]. XVI Congreso Nacional de Educación Comparada, Tenerife. https://doi.org/jbgz Carranza Esteban, R. F. \& Turpo Chaparro, J. E. (2019). Limitantes de la producción y publicación científica universitaria. Educación Médica, 20, 185-186. https:// doi.org/jbg2

Casilla, D. \& Camacho, H. (2012). Evaluación de la responsabilidad social universitaria. Opcion, 28(69), $452-465$ 
Cea Esteruelas, N. (2018). Responsabilidad socia universitaria: el papel de los medio de comunicación y sus stakeholders. Retos, 8(16), 95-105.

Céspedes Aguirre, L. (2019). Percepción de los grupos de interés de la Responsabilidad Social Universitaria en una universidad pública. Revista Conrado, 15(70), 81-89.

Chirinos Araque, Y. V. \& Pérez Peralta, C. M. (2017). La responsabilidad social universitaria: emprendimiento sostenible como impacto de intervención en comunidades vulnerables. Revista EAN, 81, 91-110. https://doi.org/jbg4

Corral Verdugo, V. (2010). Psicología de la sustentabilidad. Trillas.

Díaz Molina, M. \& Facal Santiago, S. (2011). Percepciones de los estudiantes de la Facultad de Ciencias Empresariales sobre la Responsabilidad Social Universitaria (Montevideo-Uruguay). Revista Investigación \& Desarrollo, 19(2), 340-365. https://cutt. ly/CCl8R1I

Duque, P. \& Cervantes Cervantes, L.S. (2019). Responsabilidad Social Universitaria: una revisión sistemática y análisis bibliométrico. Estudio Gerenciales, 35(153), 451-464.

Evia Alamilla, N. M., Echeverría Echeverría, R., Carrillo Trujillo, C. D. \& Quintal López, R. (2017). Ciudadanía: análisis de algunos elementos del Modelo de Responsabilidad Social Universitaria en una universidad pública. Revista CS, 23, 77-104. https:// doi.org/jbg5

Fernández Díaz, A. (2019). La interrelación instituciones universitarias - comunidad, un modelo participativo que lo promueve. Transformación, 15(1), 27-38.

Flores-Garrido, N. \& Barreto-Ávila, M. (2018). Violencia en el noviazgo entre estudiantes de la Universidad Nacional Autónoma de México. Un análisis mixto. Revista Iberoamericana de Educacion Superior, 9(25), 4261. https://doi.org/jbg6

Gaete Quezada, R. (2008). La Responsabilidad Social
Universitaria. Horizontes, 30(120), 94-127. https://doi. org/h6gf

Gaete Quezada, R. (2010). Discursos de responsabilidad social universitaria. El caso de las universidades de la macro zona norte de Chile pertenecientes al Consejo de Rectores. Perfiles Educativos, 32(128), 27-54.

Gaete Quezada, R. (2011). Relación de las universidades con sus stakeholders en Internet: un modelo de análisis. Ciencia, Docencia y Tecnología, 22(42), 9-39.

Gaete Quezada, R. (2014). La Responsabilidad Social Universitaria como política pública: Un estudio de caso. DAAPGE, 14(22), 103-128. https://doi.org/jbg7

Gaete Quezada, R. (2015a). El concepto de responsabilidad social universitaria desde la perspectiva de la alta dirección. Cuadernos de Administración, 31(53), 97-107.

Gaete Quezada, R. (2015b). El voluntariado universitario como ámbito de aprendizaje servicio y emprendimiento social. Un estudio de caso. Proyecto Juventudes, 43, 235-260.

Gaete Quezada, R. (2015c). La Responsabilidad Social Universitaria desde la perspectiva de las partes interesadas: un estudio de caso. Actualidades Investigativas en Educación, 15(1), 1-29.

Gaete Quezada, R. (2015d). Responsabilidad social en el gobierno y gestión de las universidades estatales chilenas. Civilizar, 15(29), 163-180. https://cutt.ly/ qCl7qqo

Gaete Quezada, R. (2016). La Responsabilidad Social Universitaria en la identidad corporativa de las universidades chilenas. Un análisis de contenido. DAPPGE, 16(26), 43-74.

Gálvez, A. \& Gutiérrez, I. (2018). Diagnóstico de la imagen y el posicionamiento de la Universidad Internacional de Ciencia y Tecnología [Ponencia]. https://cutt.ly/ nYB5UL4

Garbizo Flores, N., Ordaz Hernández, M. \& Lezcano Gil, A. M. (2020a). El profesor universitario ante el reto de educar: su formación integral desde la Responsabilidad Social Universitaria. Revista de 
Estudios y Experiencias en Educación, 19(40), 151-168.

\section{https://doi.org/hw4p}

Garbizo Flores, N., Ordaz Hernández, M. \& Lezcano Gil, A. M. (2020b). Relación Universidad - Comunidad: expresión de responsabilidad social de sus protagonistas. Propósitos y Representaciones, 8(2), 1-13. https://doi.org/jbg8

Gaudiano, E.J. G., Meira-Cartea, P.A. \& Martínez-Fernández, Y. C. N. (2015). Sustentabilidad y Universidad: Retos, ritos y posibles rutas. Revista de La Educacion Superior, 44(175), 69-93. https://doi.org/frw9

Giménez, E., Benítez, G., Szwako, A., Moreno, M., Burgos, J., Corbeta, R., Recalde, J. \& Torres, E. (2016). Responsabilidad Social Universitaria en el curriculum de la carrera de medicina de la FCM UNA del levantamiento de demandas y necesidades a la declaracion del perfil generico del egresado. Anales de la Facultad de Ciencias Médicas, 49(1), 105118.

Gómez, L., Alvarado, Y. \& Pujols, A. (2018). Implementing University Social Responsibility in the Caribbean : Perspectives of Internal Stakeholders. Revista Digital de Investigación en Docencia Universitaria, 12(2018), 101-120.

Huerta-Riveros, P. \& Gaete-Feres, H. (2017). Responsabilidad social universitaria através de los reportes de sostentibilidad del Global Reporting Iniative: experiencia de una universidad pública. Revista Iberoamericana de Educacion Superior, 8(23), 120-137. https://doi.org/jbg9

Ibarra Uribe, L. M., Fonseca Bautista, C. D. \& Santiago García, R. (2020). La responsabilidad social universitaria. Misión e impactos sociales. Sinéctica, Revista Electrónica de Educación, 54, 1-18. https://doi. org/jbhb

Lescher, M., Lescher, I. \& Caira Tovar, N. (2015). Responsabilidad social universitaria en Maracaibo, Venezuela. Telos: Revista de Estudios Interdisciplinarios en Ciencias Sociales, 17(2), 196-207.
López Noriega, M. D., Zalthen Hernández, L. \& Cervantes Rosas, M. (2016). La responsabilidad social universitaria desde la perspectiva del alumno. $\mathrm{Ra}$ Ximhai, 12(6), 305-314. https://doi.org/jbhc

Lúquez de Camacho, P., Fernández de Celayaran, O., Sansevero de Suarez, I. \& Fontanilla, N. (2012). Praxis de competencias ciudadanas en el ejercicio de la Responsabilidad Social Universitaria. Opcion, 28(69), $480-497$.

Madorrán-García, C. (2012). ¿Es la universidad pública española socialmente responsable? Revista Iberoamericana de Educación Superior, 3(8), 90-103. https://doi.org/jbhd

Malluk Marenco, A. \& D’Luyz Sánchez, C. (2017). Evaluación del programa Reciclando Ando en instituciones educativas y comunidades vulnerables de Montería - Colombia, en el marco de la Responsabilidad Social Universitaria. Estudio de casp. Revista Encuentros, 16(01), 46-63. https://doi.org/jbhf

Martí Noguera, J. J., Martí-Vilar, M. \& Almerich, G. (2014). Responsabilidad social universitaria: influencia de valores y empatía en la autoatribución de comportamientos socialmente responsables. Revista Latinoamericana de Psicología, 46(3), 160-168. https:// doi.org/f25v9m

Martínez de Carrasquero, C., Mavárez, R., Rojas, P. \& Carvallo, B. (2008). La responsabilidad social universitaria como estrategia de vinculación con su entorno social. Frónesis, 15(3), 1-10.

Martínez, M. J., Salom, D. G. \& Mendoza, D. M. (2019). Revisión sistemática de responsabilidad social universitaria y aprendizaje servicio. Revista Mexicana de Investigación Educativa, 24(80), 149-172. https:// cutt.ly/IYB6lww

Méndoza Avilés, H., Loayza Chavarría, M. \& González Vásquez, M. (2019). Análisis de la gestión ambiental como indicador de la responsabilidad social universitaria. Universidad y Sociedad, 11(2), 37-41.

Moreno Moreno,A. \& Aguirre Gonález, E. (2018). Diagnóstico 
sobre la práctica de responsabilidad social aplicada en la Universidad del Valle Sede Norte. Periodo 2015 2015. Cuadernos de Administración, 34(62), 66-81.

López-Noriega, M.D., Zalthen-Hernández, L. \& CarrilloMarín, A. M. (2015). Las buenas prácticas en el marco de la Responsabilidad Social Universitaria. Ra Ximhai, 11(4), 101-112. https://cutt.ly/DYNi62N

Nuñez Flores, M. I., Avelar Bribiesca, M. G. \& Ramírez Villacorta, Y. (2020). Propuesta de un diseño didáctico con tecnología para un entorno educativo rural. Zincografía, 4(7), 5-17.

Olarte-Mejía, D. V. \& Ríos-Osorio, L. A. (2015). Enfoques y estrategias de responsabilidad social implementadas en Instituciones de Educación Superior. Una revisión sistemática de la literatura científica de los últimos 10 años. Revista de La Educacion Superior, 44(175), 19 40. https://doi.org/ghdp3j

Pepe, N. C., Briceño, M. E., Carrero, M. \& Lahoud, F. (2009). Pertinencia del docente universitario. Caso de estudio: instituto universitario de tecnología del estado trujillo. Visión Gerencial, 2, 341-354.

Peralta, R. (2012). Formación académica estudiantil requerida para la praxis de la Responsabilidad Social Universitaria. Opcion, 28(69), 498-516.

Popay, J., Roberts, H., Sowden, A., Petticrew, M., Arai, L., Rodgers, M., Britten, N., Roen, K. \& Duffy, S. (1995). Guidance on the conduct of narrative synthesis in Systematic Reviews. A Product from the ESRC Methods Programme. En J Popay (Ed.). Applying the guidance: a narrative synthesis of studies of the effectiveness of interventions for promoting smoke alarm ownership and function (pp. 25-66). ESRC.

Pumacayo Palomino, I. I., Calla Vásquez, K. M., Yangali Vicente, J. S., Vasquez Tomás, M. R., Arrátia Méndez, G. K. \& Rodríguez López, J. L. (2019). Responsabilidad social universitaria y la calidad de servicio administrativo. Apuntes Universitarios, 9(2), 46-63. https://doi.org/jbhh

Ramos Parra, C. (2012). Cultura innovativa para la universidad de servicio en el marco de la responsabilidad social universitaria. Opcion, 28(68), 287-302. https://cutt.ly/qYNqm5T

Rangel Toloza, Z., Balbo de Sánchez, J., Avendaño Gelves, D. \& Acosta Rivas, H. (2012). Ética, capital social y desarrollo en la Universidad Nacional Experimental del Táchira. Opcion, 28(68), 273-286. https://cutt.ly/ $\underline{\mathrm{XYNqT7D}}$

Raza Calderón, N. A. \& Loachamín Paucar, J. J. M. (2015). La responsabilidad social universitaria: Componente fundamental en la formación de los futuros docentes de la carrera de Pedagogía, de la Universidad Politécnica Salesiana. Alteridad, 10(1), 86-99. https:// doi.org/jbhp

Rojas Valdés, A., Rovira Álvarez, Y., Fernández-Larrea, G. \& Mirabal González, Y. (2020). La formación de actores en función de la responsabilidad social comunitaria. Cooperativismo y Desarrollo, 8(1), 147-159. https://cutt. ly/OYNqJRD

Tobón Marulanda, F. Á., López Giraldo, L. A. \& Londoño Arroyave, C. D. (2019). Investigación formativa y prácticas académicas integradoras en el marco de la Responsabilidad Social Universitaria: un análisis a partir de metodología mixta. Entramado, 15(2), 188200. https://doi.org/jbhq

Tricco, A. C., Lillie, E., Zarin, W., O’Brien, K. K., Colquhoun, H., Levac, D., Moher, D., Peters, M. D. J., Horsley, T., Weeks, L., Hempel, S., Akl, E. A., Chang, C., McGowan, J., Stewart, L., Hartling, L., Aldcroft, A., Wilson, M. G., Garritty, C., ... Straus, S. E. (2018). PRISMA Extension for Scoping Reviews (PRISMA-ScR): Checklist and Explanation. Annals of Internal Medicine, 169(7), 467. https://doi.org/gfd8vk

Union de Responsabilidad Social Universitaria Latinoaméricana [URSULA] (2019a). Manual de Responsabilidad Social Universitaria. El modelo URSULA: estrategias, herramientas, indicadores. URSULA. https:// cutt.ly/wYNw5qY

Union de Responsabilidad Social Universitaria 
Latinoaméricana [URSULA] (2019b). Resultados investigación continental URSULA: Estado del arte de la RSU en América Latina 2019. URSULA. https://cutt.ly/ NYNecP7

Uribe Macías, M. E., Orjuela Ramírez, D. F. \& Moreno Barragán, X. (2016). SE de la Universidad del Tolima frente al stakeholder "proveedores." Dimensión Empresarial, 14(2), 115-128.

Vallaeys, F. (2014). La Responsabilidad social universitaria: un nuevo modelo universitario contra la mercantilización. Revista Iberoamericana de Educación Superior, 5(12), 105-117. https://doi.org/hw44

Vallaeys, F. (2016). Introducción a la Responsabilidad Social Universitaria. RSU. Universidad Simón Bolívar.

Vallaeys, F. \& Álvarez Rodríguez, J. (2019). Hacia una definición latinoamericana de responsabilidad social universitaria. Aproximación a las preferencias conceptuales de los universitarios. Educación XX1, 22(1), 93-116. https://doi.org/h6gg

Vallaeys, F. \& Carrizo, L. (2006), Responsabilidad social universitaria: marco conceptual, antecedentes, herramientas. Cd Multimedia, Lima, Banco Interamericano de Desarrollo.

Vallaeys, F., Dela Cruz, C. \& M. Sasia,P.(2009). Responsabilidad social universitaria. Manual de primeros pasos. McGrawHill.

Vásquez Ibáñez, V. (2019). La Responsabilidad Social en la Educación Superior: una revisión de la alineación entre el discurso educativo oficial y el curriculum explícito de las instituciones de educación superior de Oaxaca. Revista de La Educación Superior, 48(191), 113-137. https://doi.org/jbhx

Vega, A., Rojas, M. J. \& Salazar, G. (2018). Responsabilidad Social Universitaria: Aportes recientes al desarrollo de las empresas de menor tamaño en Chile. Revista Venezolana de Gerencia, 23(1), 328-345. https://cutt.ly/ YCzrJUq

Velasco Ramírez, S. Y., Valencia, J., Vera Porras, J. P. \& Echavarria García, J. (2017). Gestión de eficiencia energética como fiente de ventaja competitiva en la Universidad de Santiago de Cali. Revista Científica Hermes, 18, 344-381.

Vera Guadrón, L. J., Gómez Silva, M. L., Coromoto Acosta, Y. \& Perozo Piñero, L. (2012). La docencia en el marco de la responsabilidad social universitaria. Opcion, 28(68), 257-272. https://cutt.ly/XYNrtXV

Zárate-Rueda, R. \& Hernández-Suárez, M. I. (2016). Orientaciones socialmente responsables para la permanencia estudiantil en programas de educación a distancia de la Universidad Industrial de Santander - UIS. Entramado, 12(1), 250-264. https://doi.org/jbh5

Zárate Rueda, R. \& Mantilla Pinilla, E. (2014). La deserción estudiantil UIS, una mirada desde la responsabilidad social universitaria. 